International Mathematical Forum, 2, 2007, no. 54, 2655 - 2659

\title{
On Dual Versions of Krull's Intersection Theorem
}

\author{
H. Ansari-Toroghy and F. Farshadifar \\ Department of Mathematics \\ Faculty of Science, Guilan University \\ P. O. Box 1914, Rasht, Iran \\ ansari@guilan.ac.ir
}

\begin{abstract}
Let $R$ be a commutative Noetherian ring (with identity) and let $M$ be an $R$-module. In this paper we will state and prove some dual versions of Krull's intersection Theorem.
\end{abstract}

\section{Mathematics Subject Classification: 13E05, 13E10}

Keywords: Krull's intersection Theorem, codomain, Artinian modules, and secondary modules

\section{Introduction}

Throughout this paper $R$ will denote a commutative ring with an identity.

Let $R$ be a Noetherian ring and let $I$ be an ideal of $R$. A well known version of Krull's intersection Theorem states that if $M$ is a finitely generated $R$-module and if $L=\cap_{i=1}^{\infty} I^{n} M$, then $L=I L$. Another version of this Theorem says that if $R$ is a domain and $I$ is a proper ideal of $R, \cap_{i=1}^{\infty} I^{n}=0$. In this paper we state and prove the dual versions of the above mentioned results (see $3.1,3.2,3.5$, and 3.6).

\section{Auxiliary results}

\section{Definition 2.1.}

(a) An $R$-module $M$ is said to be finitely cogenerated (see [1]) if for every set $\left\{M_{\lambda}\right\}_{\lambda \in \Lambda}$ of submodules of $M, \cap_{\lambda \in \Lambda} M_{\lambda}=0$ implies $\cap_{i=1}^{n} M_{\lambda_{i}}=0$ for some positive integer $n$. 
(b) An non-zero $R$-module $M$ is said to be secondary (see [4]) if for each $\alpha \in R$, the homothety $\alpha: M \rightarrow M$ is either surjective or nilpotent.

(c) A secondary representation of an $R$-module $M$ is an expression of $M$ as a finite sum of secondary submodules of $M$ (see [4]).

(d) Let $M$ be an $R$-module. The dual notion of $Z(M)$, the set of zero divisors of $M$, is denoted by $W(M)$ and defined by

$$
W(M)=\{a \in R: \text { the homothety } M \stackrel{a}{\rightarrow} M \text { is not surjective }\} .
$$

$M$ is said to be codomain (see [2]) if $W(M)=0$.

Example 2.2. Let $p$ be a prime number. The $\mathbb{Z}$-module $\mathbb{Z}\left(p^{\infty}\right)$ (here $\mathbb{Z}$ is the ring of integers) is a codomain.

Remark 2.3. (A dual of Nakayama Lemma (see [2]).) Let $M$ be a finitely cogenerated $R$-module and let $I$ be an ideal of $R$ with $I \subseteq \operatorname{Jac}(R)$, where $\operatorname{Jac}(R)$ denotes the Jacobson radical of $R$. Further let $\left(0:_{M} I\right)=0$. Then $M=0$.

Lemma 2.4 Let $R$ be a Noetherian ring and $I$ be an ideal of $R$. If $M$ is a secondary $R$-module, then $I M=M$ or there exists a positive integer $n$ such that $I^{n} M=0$.

Proof. Suppose that $I M \neq M$ and $a \in I$. Then we have $a M \neq M$. Since $M$ is a secondary $R$-module, there exists a positive integer $k$ such that $a^{k} M=0$ so that $I \subseteq \sqrt{A n n_{R}(M)}$. Further since $R$ is a Noetherian ring, there exists a positive integer $n$ such that $I^{n} \subseteq A n n_{R}(M)$ as desired.

\section{Main results}

Throughout this section $R$ will denote a Noetherian ring.

Theorem 3.1. (Firest dual version of Krull's intersection Theorem) Let $M$ be an Artinian $R$-module and let $I$ be an ideal of $R$. Set $L=\sum_{i=1}^{\infty}\left(0:_{M} I^{i}\right)$. Then $L=\left(L:_{M} I\right)$.

Proof. If $\left(0:_{M} I\right)=0$, then the claim is clear. So we assume that $\left(0:_{M} I\right) \neq 0$. This implies that $\left(L:_{M} I\right) \neq 0$. Now by [3], $\left(L:_{M} \underline{a}\right)$ has a secondary 
representation. Let

$$
\left(L:_{M} I\right)=\sum_{i=1}^{n} S_{i}
$$

be a minimal secondary representation of $\left(L:_{M} I\right)$, where $S_{i}$ is a secondary submodule of $M$ for each $i=1,2, \ldots, n$. It is enough to show that $S_{i} \subseteq L$ for each $i=1,2, \ldots, n$. Suppose that for some $j$ with $1 \leq j \leq n$, we have $S_{j} \not L L$. Now we have

$$
I S_{j} \subseteq \sum_{i=1}^{n} I S_{i} \subseteq L .
$$

Hence $I S_{j} \subseteq L$. Since $S_{j}$ is secondary, by Lemma $2.4, I S_{j}=S_{j}$ or there exists a positive integer $t$ such that $I^{t} S_{j}=0$. If $I S_{j}=S_{j}$, then $S_{j} \subseteq L$, which is a contradiction. If $I^{t} S_{j}=0$, then $S_{j} \subseteq\left(0:_{M} I^{t}\right) \subseteq L$ which is again a contradiction. Hence $S_{i} \subseteq L$ for all $i=1,2, \ldots, n$ so that $\left(L:_{M} I\right) \subseteq L$. This completes the proof.

Corollary 3.2. Let $M$ be an Artinian $R$-module and let $I$ be an ideal of $R$ such that $I \subseteq \operatorname{Jac}(R)$. Then $\sum_{i=1}^{\infty}\left(0:_{M} I^{i}\right)=M$.

Proof. Set $L=\sum_{i=1}^{\infty}\left(0:_{M} I^{i}\right)$. By Theorem 3.1, $L=\left(L:_{M} I\right)$. This implies that $\left(0:_{M / L} I\right)=0$. Now by [1, Prop. 10.10], $M / L$ is a finitely cogenerated $R$-module. Hence by Remark $2.3, M=L$ as desired.

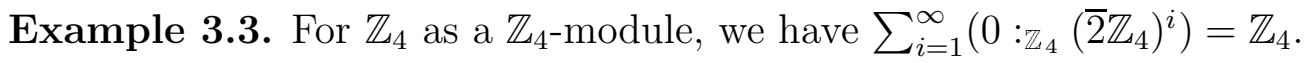

Corollary 3.4. Let $M$ be an Artinian $R$-module and let $R$ be a local ring with unique maximal ideal $I$. Then we have the following.

(a) If $N$ is a submodule of $M$, then $\sum_{i=1}^{\infty}\left(N \cap\left(0:_{M} I^{i}\right)\right)=N$.

(b) If $N$ and $K$ are submodules of $M$ such that $N \cap\left(K:_{M} I\right) \subseteq K$, then $N \subseteq K$.

Proof. (a) This is an immediate consequence of 3.2 by using the fact that

$$
\left(0:_{N} I^{i}\right)=N \cap\left(0:_{M} I^{i}\right)
$$

(b) Since $N \cap\left(K:_{M} I\right) \subseteq K$, we have

$$
\left(N:_{M} I\right) \cap\left(K:_{M} I^{2}\right)=\left(N \cap\left(K:_{M} I\right):_{M} I\right) \subseteq\left(K:_{M} I\right) .
$$

This in turn implies that

$$
N \cap\left(K:_{M} I^{2}\right) \subseteq N \cap\left(K:_{M} I\right) \subseteq K
$$


Hence for each $i$, we have $N \cap\left(K:_{M} I^{i}\right) \subseteq K$. Thus by part (a),

$$
N=\sum_{i=1}^{\infty}\left(N \cap\left(0:_{M} I^{i}\right)\right) \subseteq \sum_{i=1}^{\infty}\left(N \cap\left(K:_{M} I^{i}\right)\right) \subseteq K
$$

as desired.

Theorem 3.5. (Second dual version of Krull's intersection Theorem.) Let $M$ be a finitely generated Artinian $R$-module which is a codomain and let $I$ be a proper ideal of $R$. Then $\sum_{i=1}^{\infty}\left(0:_{M} I^{i}\right)=M$.

Proof. Set $L=\sum_{i=1}^{\infty}\left(0:_{M} I^{i}\right)$. Then by Theorem 3.1, $L=\left(L:_{M} I\right)$. This implies that $\left(0:_{M / L} I\right)=0$. Now set $S=1+I$. It is clear that $S$ is a multiplicatively closed subset of $R$ and $S^{-1} I \subseteq S^{-1} R$. Since $I$ is finitely generated, $S^{-1}\left(0::_{M / L} I\right)=\left(0:_{S^{-1}(M / L)} S^{-1} I\right)=0$ by [5, Ex. 9.13]. But $S^{-1}(M / L)$ is finitely cogenerated $S^{-1} R$-module so that $S^{-1}(M / L)=0$ by Remark 2.3. Now since $M$ is finitely generated, there exists an element $x \in R$ with $x=1+a$, where $a \in I$, such that $x(M / L)=0$. Hence $x M \subseteq L$. Since $M$ is codomain and $I$ is a proper ideal of $R, x M=M$. Thus $M=L$ as desired.

Corollary 3.6. Let $R$ be an Artinian codomain and $I$ be a proper ideal of $R$. Then $\sum_{i=1}^{\infty}\left(0:_{R} I^{i}\right)=R$.

Question 3.7. (A third dual version of Krull's intersection Theorem) Let $M$ be a finitely cogenerated $R$-module and let $I$ be an ideal of $R$. Set $L=$ $\sum_{i=1}^{\infty}\left(0:_{M} I^{i}\right)$. Is $L=\left(L:_{M} I\right)$ ?

\section{References}

[1] W. Anderson and K.R. Fuller, Rings and categories of modules, Springer-Verlag, New York-Heidelberg-Berlin 1974.

[2] H. Ansari-Toroghy and F. Farshadifar, Comultiplication modules and related results, Acta Mathematica Universitatis comenianae, submitted.

[3] I.G. Macdonald, Secondary reperesentation of modules over a commutative ring, Symp. Math. XI (1973), 23-43.

[4] H. Matsumura, Commutative ring theory, Cambr. Stud. Adv. Math. 8, Cambridge Univ. Press, Cambridge 1986. 
[5] R.Y. Sharp, Steps in commutative Algebra, London Mathematical Society Student Texts, Vol 19, Cambr. Univ. Press, 1990.

\section{Received: April 29, 2007}

